\section{Aspectos clínicos da fenilcetonúria em serviço de referência em triagem neonatal da Bahia}

\section{Clinical aspects of phenylketonuria in a reference service for neonatal screening in Bahia}

\author{
Tatiana Amorim 1 \\ Sara P.P. Gatto 2 \\ Ney Boa-Sorte 3 \\ Maria Efigênia Q. Leite 4 \\ Maria Inês M. M. Fontes 5 \\ Junaura Barretto 6 \\ Angelina X. Acosta 7
}

\section{Abstract}

Objectives: to describe clinical aspects of hyperphenylalaninemia in patients followed up on the Reference Service for Neonatal Screening (SRTN) in the state of Bahia.

Methods: cross-sectional study that enrolled all patients living in the state of Bahia and followed up on the SRTN who had a definite diagnosis of hyperphenylalaninemia prior to September, 2005. That population was made up of 46 families, with 51 patients. The analysis of data was descriptive, using measures of central tendency and dispersion.

Results: a discrete predominance of females was found (52.9\%). Most patients $(78,4 \%)$ were diagnosed on neonatal screening, thus receiving early treatment. Consanguinity was registered in $32.6 \%$ of the families. The average age at the adoption of therapy was 56.637 .8 days on the patients diagnosed on neonatal screening and 7.1 years among those with a late diagnosis.

Conclusions: the relevance of this study to public health lies upon the fact that it describes clinical features of a group of patients with a disease included on the National Neonatal Screening Program. Among clinical data, we highlight the average age at the adoption of therapy, which is higher than the recommended by medical literature, stressing the need of reinforcing the importance of early diagnosis.

Key words Phenylketonurias, Metabolism, inborn errors, Mental retardation, Neonatal screening

\section{Resumo}

Objetivos: descrever as características clínicas dos pacientes com hiperfenilalaninemia acompanhados no Serviço de Referência em Triagem Neonatal (SRTN) do estado da Bahia.

Métodos: estudo descritivo transversal, tendo como amostra todos os pacientes com diagnóstico conhecido de Hiperfenilalaninemia residentes no estado da Bahia e acompanhados no SRTN até setembro de 2005. Tal população é composta de 46 famílias, num total de 51 pacientes. A análise dos dados foi descritiva, incluindo medidas de tendência central e dispersão.

Resultados: houve discreto predomínio do gênero feminino (52,9\%). A maioria dos pacientes $(78,4 \%)$ teve seu diagnóstico estabelecido através da triagem neonatal, tendo, portanto, tratamento precoce. Consangüinidade foi registrada em 32,6\% das famílias. A média de início do tratamento entre os pacientes diagnosticados pela triagem neonatal foi de 56,6 37,8 dias, enquanto que entre os pacientes com diagnóstico tardio, foi de 7,1 anos.

Conclusões: o estudo descreve um grupo de pacientes representativo de uma patologia incluída no Programa Nacional de Triagem Neonatal (PNTN), sendo, portanto, de relevância para a saúde pública. Entre os dados clínicos, chama a atenção a média de idade do início do tratamento, superior ao recomendado na literatura, alertando para a necessidade de um maior enfoque no diagnóstico precoce.

Palavras-chave Fenilcetonúrias, Erros inatos do metabolismo, Retardo mental, Triagem neonatal 


\section{Introdução}

A fenilcetonúria (PKU) foi inicialmente descrita em 1934 pelo químico norueguês Asbjorn Fölling, através do relato de dois irmãos afetados por essa doença, e que apresentavam quadro clínico de retardo mental associado ao achado de excreção urinária de ácido fenilpirúvico e fenilacético, ${ }^{1}$ tendo sido o primeiro erro inato do metabolismo a ser oficialmente associado à presença de deficiência mental ${ }^{2}$. Trata-se de uma doença genética de herança autossômica recessiva, cujo defeito bioquímico básico consiste na deficiência da enzima fenilalanina - hidroxilase (PAH), responsável pela conversão hepática de fenilalanina em tirosina, ${ }^{3}$ enzima esta codificada por um gene localizado no cromossomo 12 (12q22-q24), já tendo sido descritas até o momento 498 diferentes mutações neste lócus. ${ }^{4} \mathrm{Na}$ década de 50, foram desenvolvidas as primeiras fórmulas alimentares isentas de fenilalanina, permitindo a substituição precoce da alimentação do lactente por uma dieta pobre nesse aminoácido, e prevenindo de maneira amplamente eficaz o retardo mental.5,6 As hiperfenilalaninemias podem ser classificadas em PKU clássica, PKU leve ou Hiperfenilalaninemia não-PKU (HPA não-PKU), a depender do nível de fenilalanina sérica ao diagnóstico, que nos fornece uma estimativa da atividade enzimática residual, permitindo o estabelecimento do fenótipo bioquímico (Tabela 1).

Tabela 1

Classificação bioquímica das hiperfenilalaninemias.

\begin{tabular}{lccc}
\hline Tipo & Fal sérica $(\mathbf{m g} \%)$ & Atividade enzimática (\%) & Tratamento \\
\hline PKU clássica & $>20$ & $<1$ & Sim \\
PKU leve & $10-20$ & $1-3$ & Sim \\
HPA não-PKU & $3,5-10$ & $>3$ & Não \\
\hline
\end{tabular}

PKU clássica = Fenilcetonúria clássica.; PKU leve = Fenilcetonúria leve; HPA não-PKU = Hiperfenilalaninemia não-fenilcetonúria. Fal = fenilalanina Fonte: Adaptado de Martins, A. Inborn errors of metabolism: a clinical overwiew. 7 dos recém-nascidos. Na Bahia, a realização do "Teste do Pezinho" teve início em 1992 na APAE de Salvador, que se tornou Serviço de Referência em 2001. O presente trabalho tem como objetivo descrever as características clínicas dos pacientes com diagnóstico de PKU ou Hiperfenilalaninemia nãoPKU acompanhados no Serviço de Referência em Triagem Neonatal (SRTN) da Bahia.

\section{Métodos}

Foram incluídos no estudo 46 famílias, com diagnóstico bioquímico de fenilcetonúria, definido como fenilalanina (Fal) sérica $\geq 10 \mathrm{mg} \%$, ou de hiperfenilalaninemia não-fenilcetonúria, definido como níveis de Fal entre 3,5 e $10 \mathrm{mg} \% .{ }^{3}$ Foram estudados todos os pacientes que mantinham o acompanhamento até setembro de 2005, incluindo pacientes com diagnóstico tardio também acompanhados no serviço de referência. Esse serviço teve sua equipe multidisciplinar ampliada e adequada às necessidades do tratamento em julho de 2001, a partir da portaria 822/01 do Ministério da Saúde,10 que estabeleceu o Programa Nacional de Triagem Neonatal (PNTN), e funciona na Associação de Pais e Amigos dos Excepcionais em Salvador, capital da Bahia, realizando o acompanhamento regular dos pacientes diagnosticados através do teste do pezinho no estado da Bahia, incluindo, além da fenilcetonúria, hipotireoidismo congênito e hemoglobinopatias. $\mathrm{O}$ atendimento é realizado por equipe multidisciplinar, composta por pediatra, geneticista, hematologista e endocrinologista pediátricos, nutricionista, nutrólogo, assistente social, psicólogo e biólogos. Dispõe de um laboratório especializado responsável pelas dosagens seriadas de Fal utilizadas como parâmetro laboratorial de controle da doença. Os pacientes são acompanhados quinzenalmente ou mensalmente, para avaliação clínico-laboratorial, acompanhamento do desenvolvimento neuropsicomotor e adequação da prescrição dietética. Todos os pacientes diagnosticados através da triagem neonatal pelo SRTN da APAE em Salvador encontram-se em acompanhamento multidisciplinar, sendo ainda o serviço referência para realizar o tratamento e acompanhamento de todos os casos de Fenilcetonúria diagnosticados no estado da Bahia.

Foram coletados dados, através de protocolo clínico, referentes a gênero, idade do diagnóstico e do início do tratamento, presença de outros afetados na família, naturalidade, ancestralidade, grupo racial, consangüinidade parental, forma do diagnóstico (triagem neonatal ou doença estabelecida clinica- 
mente), dosagem da fenilalanina ao diagnóstico e conseqüente fenótipo bioquímico e presença de sinais e sintomas no momento do diagnóstico (irritabilidade, despigmentação cutânea e de cabelos, déficit de crescimento, retardo do desenvolvimento neuropsicomotor, distúrbios de comportamento e odor urinário peculiar).

Os dados foram obtidos através de revisão de prontuários complementada por entrevistas e organizados e tabulados numa planilha eletrônica. O estudo foi descritivo transversal, sendo realizadas medidas de distribuição de freqüências, tendência central e dispersão, utilizando o programa MS-Excel para Windows ${ }^{\circledR}$ versão 2000. Os responsáveis assinaram um termo de consentimento livre e esclarecido dando permissão para a participação no estudo, que foi aprovado pelo Comitê de Ética em Pesquisa do Centro de Pesquisa Gonçalo Moniz da Fundação Oswaldo Cruz (FIOCRUZ) da Bahia.

\section{Resultados}

Foram avaliados os afetados de 46 famílias, com um total de 51 indivíduos com PKU ou HPA nãoPKU. Em todas as famílias em que havia mais de um afetado $(8,7 \%)$, este pertencia à irmandade do caso índice. De acordo com os dados da triagem neonatal na Bahia, a incidência da patologia é de aproximadamente 1:22.000 nascidos-vivos no Estado (relatório anual do SRTN, apresentado no boletim informativo anual da APAE Salvador, de circulação interna, (dados não publicados).11

Houve preponderância do gênero feminino na população estudada, com $52,9 \%$ (27) de meninas, contra $47,1 \%$ (24) de meninos.

O diagnóstico foi realizado através de triagem neonatal em 40 casos $(78,4 \%)$ e através de doença clínica (sendo a principal manifestação deficiência mental, variando de leve a grave) em 11 pacientes $(21,6 \%)$. Em quatro famílias havia mais de um afetado. Em três situações o propósito havia sido diagnosticado por triagem neonatal. Na primeira família, o irmão era um gemelar, tendo portanto diagnóstico e tratamento também precoces; na segunda família, duas irmãs afetadas foram diagnosticadas tardiamente após diagnóstico neonatal do probando; na terceira, o mesmo ocorreu com um irmão do propósito. Na quarta família, ambos os irmãos tiveram diagnóstico tardio, um aos três anos e outro aos 15 anos. Em todos esses casos, foi instituído o tratamento, com melhora significativa do quadro clínico, embora a deficiência mental já estivesse instalada.
A classificação do fenótipo bioquímico, com base nos níveis de fenilalanina ao diagnóstico, estabeleceu a PKU clássica em 37 pacientes $(72,5 \%)$, PKU leve em $8(15,7 \%)$ e HPA - não PKU em (seis) $6(11,8 \%)$ pacientes (Tabela 2$)$.

Tabela 2

Classificação quanto ao fenótipo bioquímico dos 51 pacientes com PKU do Serviço de Referência em Triagem Neonatal da Associação Pais e Amigos e Excepcionais. Salvador, Bahia.

\begin{tabular}{lrr}
\hline Fenótipo bioquímico & N & $\%$ \\
\hline PKU clássica & 37 & 72,5 \\
PKU leve & 8 & 15,7 \\
HPA-não PKU & 6 & 11,8 \\
Total & 51 & 100,0 \\
\hline
\end{tabular}

(PKU clássica $=$ Fenilcetonúria clássica; PKU leve = Fenilcetonúria leve; HPA não-PKU = Hiperfenilalaninemia não-fenilcetonúria.)

Quanto à presença de sintomas ao diagnóstico (Tabela 3), todos os pacientes com diagnóstico tardio apresentavam sintomas da doença, enquanto que entre os 34 pacientes diagnosticados precocemente (através de triagem neonatal) como afetados por PKU leve ou clássica, 44,1\% também já apresentavam sinais e sintomas, sendo os mais freqüentes a irritabilidade, caracterizada principalmente como dificuldade para dormir e se alimentar, e choro freqüente, e a despigmentação dos cabelos (explicada pela interferência na via de síntese da melanina que ocorre nos pacientes com fenilcetonúria).

\section{Tabela 3}

Presença de sintomas e sinais clínicos em 51 pacientes com PKU leve ou clássica, do Serviço de Referência em Triagem Neonatal da Associação Pais e Amigos de Excepcionais. Salvador, Bahia.

\begin{tabular}{lcccccc}
\hline Forma de diagnóstico & \multicolumn{2}{c}{ Sintomáticos } & Assintomáticos & \multicolumn{2}{c}{ Total } \\
\hline & $n$ & $\%$ & $n$ & $\%$ & $n$ & $\%$ \\
\hline Triagem neonatal & 15 & 44,1 & 19 & 55,9 & 33 & 78,4 \\
$\begin{array}{l}\text { Doença } \\
\text { Total }\end{array}$ & 11 & 100,0 & 0 & 0,0 & 11 & 21,6 \\
& 26 & & 19 & & 44 & 100,0 \\
\hline
\end{tabular}


Quarenta e quatro dos 45 pacientes diagnosticados como PKU clássica ou leve encontram-se em tratamento dietético regular. Um único paciente, embora seja acompanhado no Serviço, não tem a dieta recomendada seguida pelos genitores e, aos seis anos de idade, apresenta déficit cognitivo atribuído à doença. Um paciente com diagnóstico precoce só iniciou o tratamento aos seis meses de idade, devido a não comparecimento ao Serviço em tempo hábil, a despeito da busca ativa, em decorrência de dificuldades na viabilização do transporte pelo município de origem, e atualmente, aos 27 meses de idade, apresenta leve retardo no desenvolvimento neuropsicomotor. Os seis pacientes com diagnóstico de HPA - não PKU permanecem em acompanhamento, porém sem tratamento dietético, conforme recomenda a literatura. 3

Questionados quanto à ascendência da família, a maioria $(91,3 \%)$ não referia ascendência estrangeira, enquanto que uma família possuía antecedentes portugueses e espanhóis, uma outra, portugueses e indígenas, uma terceira, franceses, portugueses e africanos, e uma quarta, indígenas.

Os casos diagnosticados são oriundos de 22 diferentes municípios, sendo Monte Santo, na região norte do Estado, o município do interior que apresenta maior número, com quatro pacientes em acompanhamento. A capital do estado (Salvador) responde por pequena parcela do total dos pacientes acompanhados (9).

Consangüinidade entre os pais dos afetados foi detectada em 32,6\% das famílias.

Quarenta pacientes $(78,4 \%)$ foram classificados no grupo racial branco, enquanto que $19,6 \%$ eram mulatos e apenas um $(2 \%)$ foi classificado como preto. A classificação fenotípica foi realizada por profissional treinado, usando os critérios estabelecidos por Krieger. 12

O início do tratamento, entre os pacientes diagnosticados por triagem neonatal, variou de 17 dias a (seis) 6 meses de idade, com amplitude de 163 dias, média de $56,6 \pm 37,78$ dias e mediana de 45 dias. Entre os pacientes com diagnóstico tardio, a idade ao iniciar o tratamento variou de (um) 1 a (quinze) 15 anos, com média de 7,1 anos e mediana de (sete) 7 anos.

\section{Discussão}

O Serviço de Referência em Triagem Neonatal do Estado da Bahia realiza diagnóstico, busca ativa, tratamento e acompanhamento dos pacientes com PKU rastreados. Os casos diagnosticados são oriundos preferencialmente do interior do estado, onde a cobertura da triagem neonatal é mais baixa, sendo a capital (cobertura maior que $80 \%$ ), conforme relatório interno da APAE11, responsável por apenas $17,6 \%$ dos casos, a maioria tardios. Entre os 22 diferentes municípios baianos com casos diagnosticados, os municípios de Monte Santo e Uauá, vizinhos e localizados no norte do estado, e que realizam triagem neonatal de maneira efetiva há poucos anos, mostram concentração de pacientes em acompanhamento. Com respeito a esses municípios, várias hipóteses podem ser aventadas, incluindo efeito fundador e consangüinidade, de modo que um projeto envolvendo rastreamento em massa, objetivando incluir também os casos tardios, está sendo desenvolvido para a investigação da PKU nessa região. A pequena freqüência da patologia na capital pode se dever a diferenças na composição populacional (colonização) entre o litoral e o interior da Bahia. Dados produzidos pelo estudo das mutações causadoras de doença nos pacientes poderão ajudar a esclarecer tais questões. Na Bahia, dados do DATASUS estimam em cerca de 20.000 o número médio aproximado de nascimentos /mês no estado. 13

O SRTN tem realizado aproximadamente 16.000 exames/mês, o que corresponde a uma cobertura de cerca de $80 \%$ dos nascimentos na Bahia. Tal número torna a presente amostra representativa da população, no que se refere aos casos detectados por triagem neonatal, configurando (considerando-se os casos rastreados a partir de julho de 2001 - data da portaria do Ministério da Saúde), 10 uma incidência de um caso de PKU para cada 22.000 nascidosvivos. Esses números mostram uma frequiência superior à esperada, tomando-se por base populações afro-descendentes. ${ }^{1}$ Já o diagnóstico tardio depende de outros fatores, como o conhecimento dos profissionais de saúde sobre a patologia e o acesso da população aos serviços de diagnóstico. Com o aumento progressivo da cobertura do "Teste do Pezinho" ao longo dos 13 anos de sua implantação na Bahia, o número de casos vem aumentando e não houve perda de famílias rastreadas, uma vez que é realizado um processo rigoroso de busca ativa. A grande preponderância da triagem neonatal como método diagnóstico, ao tempo em que denota uma melhoria importante no âmbito da saúde pública, uma vez que o diagnóstico e tratamento precoces previnem amplamente o desenvolvimento dos sintomas, em especial o retardo mental, chama também a atenção sobre o provável sub-diagnóstico da patologia. A PKU é uma doença de difícil suspeição clínica, o que se deve em grande parte à falta de conhecimento dos médicos sobre a condição, que é 
muitas vezes confundida com autismo, Síndrome de Angelman, transtorno de hiperatividade com déficit de atenção, entre outras. ${ }^{14}$ Assim, sendo a população do estado da Bahia estimada em aproximadamente 13.552.649 habitantes (DATASUS, Ministério da Saúde, 2004, dados baseados no Censo 2000), 15 estima-se que a real prevalência da Fenilcetonúria na Bahia seria de cerca de 589 afetados, o que reforça a ocorrência de significante sub-diagnóstico.

$\mathrm{O}$ maior número de pacientes do gênero feminino, observado neste estudo, não tem embasamento teórico-científico, e pode dever-se às limitações impostas pelo tamanho amostral.

O fenótipo bioquímico, estabelecido pela dosagem de Fal ao diagnóstico, nos permite inferir a atividade enzimática residual e, portanto, a resposta à dietoterapia. Entretanto, tal correlação necessita do suporte do conhecimento das mutações causadoras de doença em cada caso,16-18 estudo esse já em andamento neste mesmo Serviço, em parceria com a FIOCRUZ da Bahia.

A presença de recorrência na irmandade do propósito está de acordo com o padrão de herança autossômico recessivo, assim como a alta freqüência de consangüinidade entre os pais. Vale ressaltar que a investigação de irmãos com sintomas sugestivos permitiu o esclarecimento diagnóstico de crianças com deficiência mental de etiologia desconhecida, possibilitando ainda a instituição do tratamento, com bons resultados, ainda que não tenha sido mais possível reverter a deficiência cognitiva. 19,20

A informação de ausência de ancestralidade européia apresenta pouca confiabilidade, uma vez que a grande maioria dos pais não era capaz de definir seus antecedentes além da terceira geração. A maior prevalência de pacientes classificados como brancos pode sugerir ancestralidade européia/ caucasóide, o que estaria de acordo com o conhecimento disponível sobre as bases genéticas da fenilcetonúria. 9

Mesmo algumas crianças com diagnóstico precoce já apresentavam sinais e sintomas da patologia. Embora o quadro clássico da PKU não tratada classicamente só se torne evidente a partir dos seis meses de vida, estudos mostram que os sintomas mais sutis, tais como a irritabilidade e a tonalidade clara dos cabelos, podem ser bem mais precoces, sendo, entretanto, reversíveis, sem dano neurológico, quando o tratamento é instituído antes dos seis meses de idade. ${ }^{21-24} \mathrm{~A}$ idade média de início de tratamento foi de cerca de dois meses, considerada satisfatória, embora o objetivo seja o início ainda mais precoce da dieta. O desvio-padrão de cerca de 38 dias - deste modo próximo à média reflete o fato de ainda haver pacientes com demora significativa em ter o seu tratamento oportunizado. Muito embora o SRTN da Bahia funcione em padrões de excelência no que se refere ao diagnóstico laboratorial, busca ativa, atendimento multidisciplinar, tratamento e seguimento dos pacientes, a efetividade do programa tem sido comprometida por fatores anteriores à chegada do material do paciente (sangue em papel filtro) ao laboratório. O maior obstáculo encontrado pelo SRTN parece residir na falta de informação da população leiga e também dos profissionais de saúde, sobre a importância da coleta do "Teste do Pezinho" preferencialmente na primeira semana de vida, como fator imprescindível na operacionalização do programa, permitindo diagnóstico e tratamento precoces e a adequada prevenção do retardo mental. Tal falha se reflete em coleta tardia e, no caso de pacientes do interior do estado, demora no comparecimento da criança ao SRTN para iniciar o tratamento, o que, a longo prazo, poderá comprometer o resultado do tratamento, em termos de capacidade cognitiva.

O conhecimento dos dados locais, incluindo a incidência real da patologia, associado a um seguimento regular dos casos, irá permitir o aperfeiçoamento dos métodos e técnicas de diagnóstico, tratamento e prevenção. A realização do presente estudo chama a atenção especialmente para a importância da coleta do "teste do pezinho" em tempo hábil (idealmente entre o terceiro e o sétimo dia de vida), viabilizando o adequado início do tratamento e a prevenção da deficiência mental.

\section{Agradecimentos}

À colaboração em parceria do SRTN/APAE, em Salvador, em especial às técnicas Edith Roza, Inês Souza, Joseane Rodrigues, Patrícia Lessa e Simone Badaró, e aos biólogos Antônio Purificação e Sidney Prates, pela valiosa contribuição no levantamento dos dados para este estudo, e apoio constante durante a sua realização. 


\section{Referências}

1. Wilcox W, Cederbaum S. Amino acid metabolism. In: Emery and Rimoin's Principles and practice of medical genetics. London: C. Livingstone; 2001: p. 2405-40.

2. Kahler S, Fahey M. Metabolic disorders and mental retardation. Am J Med Genet 2003; 117C: 31-41.

3. Cederbaum S. Phenylketonuria: an update. Cur Opin Pediatri 2002; 14: 702-6.

4. Phenylalanine hydroxylase [on line] Disponível em URL: <http://www.pahdb.mcgill.ca/> [2005 May 11].

5. Centerwall S, Centerwall W. The discovery of phenylketonuria: the story of a young couple, two retarded children, and a scientist. Pediatrics 2002; 105: 120-36.

6. Burgard P. Development of intelligence in early treated phenylketonuria. Eur J Pediatr 2000; 159 (Suppl 2): S74-9.

7. Martins A. Inborn errors of metabolism: a clinical overwiew. Rev Paul Med 1999; 117: 251-65.

8. Guthrie R, Susi A. A simple phenylalanine method for detecting phenylketonuria in large populations of newborn infants. Pediatrics 1963; 32:338-43.

9. Acosta A. Análise molecular do gene da fenilalanina hidroxilase em pacientes com fenilcetonúria [tese doutorado]. Ribeirão Preto: Departamento de Genética, Faculdade de Medicina da Universidade de São Paulo; 2000.

10. Ministério da Saúde, Portaria GM/MS n. 822 de 6 de junho de 2001. Institui, no âmbito do Sistema Único de Saúde, o Programa Nacional de Triagem Neonatal / PNTN. Brasília (DF): 2001.

11. APAE (Associação de Pais e Amigos dos Excepcionais). Relatório anual. Salvador, 2004.

12. Krieger H, Morton NE, Mi MP, Azevêdo E, Freire-Maia A, Yasuda N. Racial admixture in Northeastern Brazil. Ann Hum Genet 1965; 29: 113-25.

13. Ministério de Saúde. Nascidos vivos - Bahia. Nascimentos por residência por ano do nascimento segundo município [on line] 2000-2002. Disponível em URL: http://tabnet.datasus.gov.br/cgi/tabcgi.exe?sinasc/cnv/nvB A.def. [2004 Out 28].

14. Dan B, Christiaens F, Mewasingh L, De Laet C, Goyens P. Late-treated phenylketonuria mimicking Angelman syndrome. Am J Med Genet 2001; 104: 345-6.
15. Ministério de Saúde. População residente - Bahia. População residente por ano segundo município [on line] 2004. Disponível em URL: http://tabnet.datasus.gov.br/cgi/tabcgi. exe?ibge/cnv/popba.htm. [2004 Out 28]

16. Desviat L, Pérez B, García M, Martínez-Pardo M, Baldellou A, Arena J, Sanjurjo P, Campistol J, Couce M, Fernández A, Cardesa J, Ugarte M. Relationship between mutation genotype and biochemical phenotype in a heterogeneous Spanish phenylketonuria population. Eur J Hum Genet 1997; 5: 196-202.

17. Enns G, Martinez D, Kuzmin A, Koch R, Wakeem C, Woo S, Eisensmith R, Packman S. Molecular correlations in phenylketonuria: mutation patterns and corresponding biochemical and clinical phenotypes in a heterogeneous Californian population. Pediatr Res 1999; 46: 594-602.

18. Güttler F, Guldberg P. Mutation analysis anticipates dietary requirements in phenylketonuria. Eur J Pediatr 2000; 159 (Suppl 2): S150-3.

19. Koch R, Moseley K, Ning J, Romstad A, Guldberg P, Guttler F. Long-term beneficial effects of the phenylalanine-restricted diet in late-diagnosed individuals with phenylketonuria. Mol Genet Metab 1999; 67: 148-55.

20. Levy H. Comments on final intelligence in late treated patients with phenylketonuria. Eur J Pediatr 2000; 159 (Suppl 2): S149.

21. Chang PN, Gray RM, O'Brien LL. Patterns of academic achievement among patients treated early with phenylketonuria. Eur J Pediatr 2000. 159 (Suppl 2): S96-9.

22. Griffiths P, Tarrini M, Robinson P. Executive function and psychosocial adjustment in children with early treated phenylketonuria: correlation with historical and concurrent phenylalanine levels. J Intellect Disabil Res 1997; 41: 31723.

23. Huijbregts S, de Sonneville L, Licht R, van Spronsen F, Sergeant J. Short-term dietary interventions in children and adolescents with treated phenylketonuria: effects on neuropsychological outcome of a well-controlled population. J Inherit Metab Dis 2002; 25: 419-30.

24. Waisbren SE. Comments on cognition and intelligence in phenylketonuria. Eur J Pediatr 2000; 159 (Suppl 2): S80-1.

Recebido em 19 de janeiro de 2005

Versão final apresentada em 15 de agosto de 2005

Aprovado em 16 de outubro de 2005 